\title{
Local Sufficient Rate Constraints for Guaranteed Capacity Region in Multi-Radio Multi-Channel Wireless Networks
}

\author{
Hongkun Li, Yu Cheng \\ Department of Elec. \& Comp. Eng. \\ Illinois Institute of Technology \\ \{hli55, cheng\}@iit.edu
}

\author{
Peng-Jun Wan \\ Department of Computer Science \\ Illinois Institute of Technology \\ wan@cs.iit.edu
}

\author{
Jiannong Cao \\ Department of Computing \\ Hong Kong Polytechnic University \\ csjcao@comp.polyu.edu.hk
}

\begin{abstract}
It is very challenging to compute the capacity region of a multi-radio multi-channel (MR-MC) network, which involves complex resource contention including the co-channel interferences and radio interface contentions. In this paper, we study the local sufficient rate constraints that can be constructed at each network node in a distributed manner to ensure a feasible flow allocation for the MR-MC network. The analysis of capacity region with the rate constraints is facilitated by our tool of multi-dimensional conflict graph (MDCG) [12], which systematically describes all kinds of conflict relationships in an MR-MC network. Specially, we establish two types of local sufficient constraints, the neighborhood constraint and the sufficient clique constraint, respectively; and both types can ensure a constant portion of the optimal capacity region, termed as capacity efficiency ratio. The capacity efficiency ratios associated with the neighborhood constraint and the sufficient clique constraint are related to the analysis of the interference degree and the imperfection ratio of an MDCG, respectively. A specific challenge is that methodology computing the interference degree and the imperfection ratio of single-radio single-channel (SR-SC) networks could not be directly extended to the MRMC context, because MR-MC network has disruptively different geometric properties compared to the SR-SC network: In an MRMC network, the geometric closeness does not necessarily imply interference due to possible parallel transmissions over different radios and channels. The fundamental contributions of this paper are the theoretical studies of the interference degree and the imperfection ratio of an MDCG, revealing how such graphical characteristics are related to those in the SR-SC context under the impact of the MR-MC geometric property. We also present extensive numerical results to demonstrate the effectiveness of the proposed local sufficient constraints in ensuring a larger capacity region compared to the well-known results in [1].
\end{abstract}

\section{INTRODUCTION}

Multi-radio multi-channel (MR-MC) wireless network offers higher network capacity than its single radio single channel (SR-SC) counterpart. However, it is difficult to compute the optimal network capacity or maximum feasible flow rates due to the high complexity in resolving resource contentions. A set of flow rates is feasible if there exists an interferencefree schedule policy that can achieve the desired rates. In this paper, we aim at constructing the sufficient condition with

This work was supported in part by NSF grants CNS-0832093, CNS1053777, CNS-0831831 and CNS-0916666. a guaranteed efficiency ratio to determine whether a set of flow rates is feasible in MR-MC networks. We are particularly interested in those sufficient constraints that can be constructed and verified in localized and distributed manner.

The link conflict graph [9] is used to describe the interference relations in SR-SC networks. In an MR-MC network, not only the co-channel contention but also the radio interface competence would lead to interference. The link conflict graph is not enough to describe all these interference relationships in the MR-MC context. In [12], we develop a multi-dimensional conflict graph (MDCG) to systematically describe the conflict relationships in MR-MC networks. Based on a multicommodity flow (MCF) formulation over the MDCG, the maximal independent set (MIS) constraint is sufficient and necessary in ensuring an optimal capacity region. However, searching all MISs requires global information and is computationally expensive, and thus we look for the constraints that are sufficient and can be constructed easily in the distributed fashion. The distributed implementation ensures that each network node can individually formulate and verify the sufficient constraint with local information. Furthermore, we target at those sufficient conditions with guaranteed performance that can ensure a constant portion of the optimal capacity region, defined as capacity efficiency ratio.

The first sufficient constraint is referred to as the neighborhood constraint, which just requires the knowledge of the interference neighbors of a node under consideration. The neighborhood constraint defines that a set of flow rates is feasible if the total normalized channel utilization within an interference neighborhood does not exceed 1 . The capacity efficiency ratio of neighborhood constraint is closely related to the interference degree of the MDCG, which has not been systematically studied. We reveal the relationship between the interference degree of an MR-MC network and its SRSC counterpart. Note that the capacity region under the neighborhood constraint may be over-restrictive, since it fails to consider the possible concurrent transmissions within the interference area when the tagged node is turned off [3].

We therefore seek a different kind of constraint, which defines a larger capacity region. We resort to the clique con- 
straint, since it could describe the interference relation within an interference area more accurately. The clique constraint states that the total normalized utilization of all links over a maximal clique (instead of over an interference neighborhood) is at most 1 . However, the clique constraint is a necessary condition defining an upper bound of the capacity region [9], because it only considers the conflicts within a maximal clique, but neglects the conflict relation between different maximal cliques.

It has been shown [3] that in the SR-SC context we could reach a sufficient condition by scaling down the clique constraint with a constant, termed as sufficient clique constraint. Essentially, the constant is the inverse of the imperfection ratio of the conflict graph, defined as the supremum of the ratio between the chromatic number and the clique number of the conflict graph. Unfortunately, it is difficult to compute the imperfection ratio in the MR-MC context due to its disruptively different geometric property compared to the SRSC network. In the SR-SC environment, the geometric distance is the only factor determining the conflict relation; while in the MR-MC context, two links may be active simultaneously through different radio interfaces on different channels even if they are located within each other's interference range. Such a fact implies that geometric distance is not the unique factor determining the conflict relationships in MR-MC networks. Thus, the method [19] computing the imperfection ratio of SR-SC networks, which is based on the unit distance graph (UDG) analysis, is not valid anymore for MR-MC networks.

We develop a novel technique to compute the imperfection ratio of MDCG, addressing the fundamental issue incurred by the MR-MC geometric property. The basic idea is to compute the imperfection ratio through a radical analysis of the chromatic number and the clique number of an MDCG. We construct two types of cliques in an MDCG considering the cochannel interference and radio contention respectively, and theoretically compute their sizes, which can be interpreted as the lower bound of clique number (the size of maximum clique). Regarding the chromatic number, we start from a feasible SRSC schedule, and divide a time slot into multiple sub-slots to ensure an interference-free schedule in MR-MC context. Each link can schedule the co-channel transmissions or radiocompetence transmissions in different sub-slots. The number of total required sub-slots can be viewed as an upper bound of the least required time slots (chromatic number) in MR-MC networks. For more accurate estimation, we further explore the potential parallel transmissions through different channels within a maximal clique. We achieve interesting findings that the scaled constant is closely related to that in SR-SC context, and under certain conditions they are exactly the same in spite of the MR-MC geometric property. Extensive numerical results show that the clique based sufficient condition defines a larger capacity region than that of state-of-art [1].

The reminder of this paper is organized as follows: Section II reviews more related works. In Section III, we introduce the system model and review the construction of MDCG; Section IV presents the neighborhood constraint. The clique based sufficient condition is developed in Section V. Section VI illustrates some numerical results for performance evaluation. Section VII gives the concluding remarks.

\section{RELATED WORK}

The capacity of SR-SC networks has been well studied. Asymptotic analysis is presented in [13], [14], showing that the per-node throughput will decrease with the growing number of nodes. In [9], the maximal independent set (MIS) and clique constraints are formulated considering the interference relationships. MIS constraints is sufficient and necessary condition leading to the optimal capacity if all MISs are involved. $O p$ timal scheduling policy is determined as well. Each maximal independent set takes turns in grabbing the channel for data transmission, with the proportion of transmission time for each MIS. However, searching all MISs requires global information and is computationally expensive. On the contrast, construction of the clique constraint requires only local information and can be formulated with distributed algorithm. However, it is only necessary condition defining an upper bound. The clique based sufficient condition is developed in [3] by scaling the clique constraint with a constant, which is shown as the inverse of the imperfection ratio of link conflict graph.

For the capacity of MR-MC networks, the impact of number of interfaces and channels is addressed in [15], and the asymptotic result is presented to reveal the relationship between the network capacity and the number of interfaces and channels. The optimal formulation naturally introduces the integer variable to describe the channel assignment and radio binding information. The state-of-arts [1], [2] mainly rely on the mixed integer programming (MIP) model to formulate the optimal resource allocation problem. To derive an interferencefree scheduling, the following equation must be satisfied for each link $l$ :

$$
X_{l, \tau}+\sum_{l^{\prime} \in I(l)} X_{l^{\prime}, \tau} \leq c(q) .
$$

where binary variable $X_{l, \tau}=1$ indicates that link $l$ is active in time slot $\tau . I(l)$ is the set of links conflicting with link l. $c(q)$ is a constant depending on the interference model. The constraint (1) is a necessary condition. If $c(q)$ is replaced by 1 , then it becomes the sufficient condition. There are two limitations in this formulation: solving MIP is NP-hard; More important, it is too conservative since all links within the area $I(l)$ are assumed to mutually interfere even if link $l$ is turned off. In this paper, we formulate the linear programming based on MDCG to describe all conflict relationships instead of the integer programming to compute the optimal capacity.

\section{SySTEM MODEL}

The wireless network is modeled as a directed graph $G(\mathcal{N}, \mathcal{L})$ with node set $\mathcal{N}$ and link set $\mathcal{L}$, and $|\mathcal{N}|,|\mathcal{L}|$ be number of nodes and links, respectively. We use $l_{u v}$ or $(u, v)$ to denote a directed link from node $u$ to node $v$. Consider that the whole spectrum available to the network is divided into $C$ frequency channels, represented as set $\mathcal{C H}$. We use 
$w_{u v}^{c}$ to denote the physical transmission capacity of link $(u, v)$ on channel $c$. For ease of presentation, we assume all links have the same capacity $w$ when presenting the new sufficient constraint. Moreover, let $M_{u}$ denote the number of radio interfaces available at node $u$, and $I=\max \left\{M_{u}, \forall u \in \mathcal{N}\right\}$. At any given time, an interface can tune to only one channel, but it can dynamically switch to different channels at different time slots. Define that each commodity is associated with a source destination pair $(\nu, \eta)$, and let $\Lambda$ denote the set of all source/destination pairs.

We adopt a more realistic interference model rather than the widely used protocol interference model or physical interference model [13]. This mode reflects the situation in 802.11, which requires both sender and receiver to be free of interference. Specifically, there would be conflict between link $l_{u v}$ and $l_{w z}$ if the distance $d_{a b} \leq R_{i n}$ for $a b=u w, u z, v w, v z, w u, z u, w v$ or $z v$, where $R_{i n}$ is the interference range.

\section{A. Multi-Dimensional Conflict Graph}

We make use of multi-dimensional conflict graph (MDCG) to describe the conflict in an MR-MC network. The basic idea is that the MR-MC networks has a multi-dimensional resource space, defined by radio interfaces, links, and channels. The MDCG is to describe the conflict relationship among the resource points, each represented as a radio-link-channel tuple. Specifically, a RLC tuple $p$ is defined in the format:

$$
\text { Radio-link-channel tuple: }\left(\left(x_{u}, x_{v}\right),(u, v), c\right) \text {. }
$$

The tuple indicates that the link $(u, v)$ operates on channel $c$, involving the radio interfaces $x_{u} \in M_{u}, x_{v} \in M_{v}$ at the sending node $u$ and receiving node $v$, respectively. Thus, a link $(u, v)$ can be mapped to $M_{u} \times M_{v} \times C$ RLC tuples in the MDCG. Let $\mathcal{P}$ denote the tuple set containing all tuples in an MDCG. Given the number of links $|\mathcal{L}|$ in original graph, the number of tuples in an MDCG is upperbounded by $I^{2} C|\mathcal{L}|$, in this case every node is equally equipped with $I$ radios.

To identify the conflict relations between two tuples, we define three types of events:

(E) Two different RLC tuples are associated with nodes being located within each other's interference range, according to the interference model.

(F) Two different RLC tuples are associated with the same channel.

(G) Two different RLC tuples share common radio interfaces at one or two nodes.

Two tuples conflict with each other if the condition $E F \bar{G} \cup$ $G$ is true. In particular, the condition $E F \bar{G}$ indicates that cochannel transmissions within the interference range conflict with each other, termed as interference conflict relationship. The condition $G$ indicates the interface conflict relationship that a radio interface can not support multiple transmissions (which might be associated with different channels) at a moment. Note that the interface conflict is a special issue induced by the MR-MC networking. For any tuple $p$, we define a tuple set $N(p)$, which contains those tuples interfering with

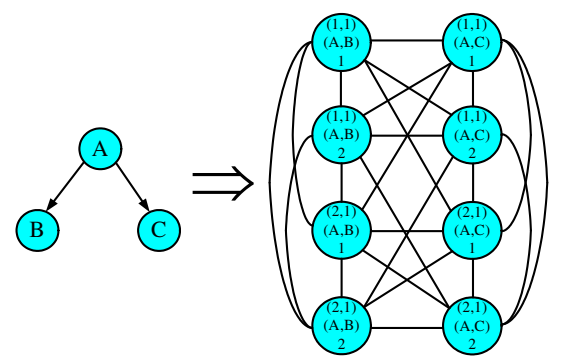

Fig. 1. Construction of MDCG.

$p$. For convenience, we assume that tuple $p$ itself is in the set $N(p)$.

We use an example to illustrate how to construct the MDCG, as shown in Fig. 1. The left side of Fig. 1 shows a small network consisting of two directed links, where node $A$ has two radio interfaces, and nodes $B$ and $C$ each have one interface. There are 2 available channels. Thus, both links $(A, B)$ and $(A, C)$ can be mapped to $2 \times 1 \times 2=4 \mathrm{RLC}$ tuples, respectively. For instance, the tuple $((2,1),(A, B), 2)$ indicates that the transmission from node $A$ to node $B$ uses the radio interface 2 at node $A$ and radio interface 1 at node $B$ through channel 2. All possible conflict relations can then be identified (according to the condition $E F \bar{G} \cup G$ ) to construct the MDCG, as shown in the right side of Fig. 1.

\section{B. MIS-Based Computing}

In the recent work [12], the maximal independent set (MIS) constraint is formulated based on the MDCG to compute the optimal capacity. Assume there are totally $K$ MISs. Let $\alpha_{m}\left(0 \leq \alpha_{m} \leq 1\right)$ denote the fraction of time allocated to the independent set $\mathcal{I}_{m}(m=1, \cdots, K)$. Considering that only one maximal independent set can be active at a time, we have the following two constraints:

$$
\begin{aligned}
& \sum_{m=1}^{K} \alpha_{m} \leq 1 \\
& \sum_{(\nu, \eta) \in \Lambda} f_{(\nu, \eta)}(p) \leq \sum_{m: p \in \mathcal{I}_{m}} \alpha_{m} w_{l(p)}^{c(p)}, \forall p \in \mathcal{P}
\end{aligned}
$$

where $l(p)$ denotes the link connection associated with tuple $p, c(p)$ is the operating channel of tuple $p$, and $f_{(\nu, \eta)}(p)$ is the amount of flow $(\nu, \eta)$ over tuple $p$. The factors $\alpha_{m}$ represents the transmission time assigned to MIS $\mathcal{I}_{m}$. However, searching all MISs is computationally expensive and requires global information. In this paper, we establish two sufficient conditions, which can be formulated in localized and distributed manner.

\section{Neighborhood CONSTRAint}

We first present the neighborhood constraint as the sufficient condition to determine the feasibility for a set of flow rates. It is claimed that for each tuple $p$, the total amount of flow rates allocated to all tuples in the tuple set $N(p)$ cannot exceed the link capacity (note that $p$ itself belongs to $N(p)$ ). In other words, we take the conflicts around the neighborhood of each tuple into account, and that is why it is named as neighborhood 
constraint. We summarize the mutual conflict relation in a $n \times$ $n$ matrix $\mathcal{H}$ as follows:

$$
\mathcal{H}_{i j}= \begin{cases}1, & \text { tuple } \mathrm{i} \text { and } \mathrm{j} \text { interfere with each other } \\ 0, & \text { otherwise }\end{cases}
$$

Note that $\mathcal{H}_{i j}$ is 1 if $i=j$, and $n$ is the number of tuples. It is obvious that the $r$ th row reveals all conflicting tuples of the $r$ th tuple. We have the following theorem:

Theorem 1: For an MR-MC network, there is a feasible schedule for a flow allocation $\mathcal{F}$, if

$$
\mathcal{H} \mathcal{F} \leq \mathcal{W}
$$

Where $\mathcal{W}$ is the capacity vector with all entries equal to channel capacity $w$, and $\mathcal{F}$ of size $n \times 1$ is the flow rate allocated to each tuple.

Due to the limited space, we skip the proof details. The proof idea is to construct a feasible schedule policy for any flow assignment satisfying condition (6). The details are similar to the proof of Theorem 4 in Section V.

The neighborhood constraint can be evaluated in a localized and distributed manner by any tuple $p$, with the knowledge of the interfering set $N(p)$ and the flow rates assigned to the tuples in $N(p)$. Note that constraint (6) is over-restrictive since it neglects the possible simultaneous transmissions over different tuples in $N(p)$ if tuple $p$ is turned off. We thus define the conflict degree $\xi(p)$ of tuple $p$, indicating the maximum number of tuples in $N(p)$ that could be active at the same time if $p$ is off. The conflict degree $\xi$ of an MDCG is computed as $\max \{\xi(p), \forall p \in \mathcal{P}\}$. The interference degree $\mathcal{K}$ is defined and studied in the SR-SC context [17]. We would like to establish the relation between $\mathcal{K}$ and $\xi$, and find the efficiency ratio of neighborhood constraint.

Theorem 2: For an MDCG and the link conflict graph from a common network topology, $\xi=\mathcal{K}+2$. Further, the neighborhood constraint (6) has an efficiency ratio of $\frac{1}{\xi}$ (i.e., $\frac{1}{\mathcal{K}+2}$ ). That means sufficient condition (6) achieves a capacity region, which is no smaller than $\frac{1}{\mathcal{K}+2}$ times the optimal region.

Proof: Without loss of generality, we randomly pick a tuple $p$. Recall that $l(p)$ denotes the link connection associated with tuple $p, c(p)$ is the operating channel of tuple $p$. According to the definition of $\mathcal{K}$, if $p$ is turned off, there are at most $\mathcal{K}(l(p))$ links that can be activated on channel $c(p)$ without interference. In addition, blocking $p$ could release its two radios and allow at most two tuples in $N(p)$ active over two different channels other than $c(p)$. Therefore, there are at most $\mathcal{K}(l(p))+2$ tuples in $N(p)$ that can be turned on if $p$ is off. That is, $\xi(p)=\mathcal{K}(l(p))+2$, and thus $\xi=\mathcal{K}+2$.

Considering the efficiency ratio of condition (6), we focus on an arbitrary tuple $p$, and assume that $f(p)$ is non-zero and the flow rates satisfy the equation $\sum_{p \in N(p)} f(p)=w$, which means this flow assignment is feasible. In fact, the maximum amount of feasible flow rate assigned to the tuples in $N(p)$ is $\xi(p) \cdot w$. Because according to the definition of conflict degree $\xi(p)$, there are up to $\xi(p)$ tuples that can be active simultaneously if $p$ is shut down. For $p$, the efficiency ratio is

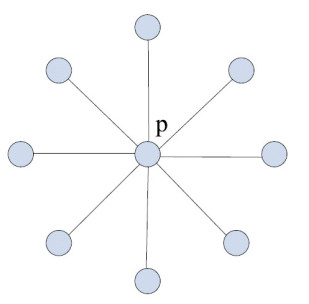

Fig. 2. Illustrative example for neighborhood constraint.

$\frac{w}{\xi(p) w}=\frac{1}{\xi(p)}$. Since $p$ is randomly selected, the constraint (6) could achieve an efficiency ratio of $\frac{1}{\xi}$.

We would like to give an example to argue that neighborhood constraint may be over-conservative. Fig. 2 illustrates an instance of MDCG (i.e., the link denotes the interference relation between two tuples). For the centered tuple $p$, we have the following constraint:

$$
f(p)+\sum_{p^{\prime} \in N(p)} f\left(p^{\prime}\right) \leq w
$$

If the centered tuple $p$ is assigned with flow rate $w$, all other tuples on the circle cannot be allocated with any flow for a feasible schedule. The total flow rates is $w$. In fact, the maximum feasible flow rates is $8 w(\xi(p)=8)$. If $p$ is turned off, every tuple around $p$ can be active with flow rate of $w$. In theory, if the number of tuples around $p$ goes to infinity (i.e., $\xi(p) \rightarrow \infty$ ), the performance of neighborhood constraint is very bad.

\section{Scaled Clique Constraint}

Though neighborhood constraint is easy to construct and verify, it may lead to considerable capacity loss. We alternatively use clique constraint to formulate a sufficient condition, since it considers the possible parallel transmissions within the interference neighborhood.

\section{A. Clique Constraint}

A clique $\mathcal{C}$ is a subset of vertices that mutually conflict in the graph. Note that each clique consists of multiple tuples in MDCG. A clique is maximal only if there is no additional tuple interfering with all tuples already in that clique. Assume we have $\mathrm{Q}$ maximal cliques, denoted as $\mathcal{C}_{1}, \mathcal{C}_{2}, \cdots, \mathcal{C}_{Q}$. Considering that the total usage of tuples within a maximal clique is at most 1, we formulate the clique constraint as follows:

$$
\sum_{p \in \mathcal{C}_{q}} \frac{1}{w_{l(p)}^{c(p)}} \sum_{(\nu, \eta)} f_{(\nu, \eta)}(p) \leq 1, \forall q=1,2, \cdots, Q
$$

Clique constraint is necessary, but not sufficient for flow allocation since it considers the interference within only one maximal clique, while neglecting the conflicts among different maximal cliques. A well-known example is the conflict graph shaped like a pentagon. We assume the capacity of each physical link is fixed to $w$ in the remainder of this paper. In SR-SC context, the clique constraints suggest a valid flow of $0.5 w$ on each link, in reality at most $0.4 w$ is achievable through the MIS-based computation [9]. We aim to find the 
TABLE I

NOTATIONS USED IN SECTION VI.

\begin{tabular}{|c|l|}
\hline $\mathrm{G}$ & The original connectivity graph \\
\hline $\mathrm{CG}$ & The conflict graph of G in SR-SC context \\
\hline MDCG & $\begin{array}{l}\text { The multi-dimensional conflict graph of G } \\
\text { in MR-MC context }\end{array}$ \\
\hline$\Delta(G)$ & Maximum node degree of graph $\mathrm{G}$ \\
\hline$\chi(G)$ & The chromatic number of graph $\mathrm{G}$ \\
\hline$\kappa(G)$ & The clique number of graph $\mathrm{G}$ \\
\hline$\delta(G)$ & $\begin{array}{l}\text { The minimum clique size of all maximal } \\
\text { cliques in graph G }\end{array}$ \\
\hline $\mathcal{Q}$ & Clique matrix \\
\hline $\mathcal{F}$ & Flow assignment vector \\
\hline$T_{\min }$ & $\begin{array}{l}\text { Minimum required time slots ensuring in- } \\
\text { terference free schedule }\end{array}$ \\
\hline
\end{tabular}

sufficient condition based on clique constraint, which can be constructed and verified in distributed may.

\section{B. Sufficiency by Scaled Clique Constraint}

It has been shown [3] that if scaling the clique constraint (8) with a constant, we could reach the sufficient condition, termed as sufficient clique constraint. Our mission is to develop new methodology to compute the constant in the MR-MC context. Throughout this section, we assume that all nodes have the same number of radio interfaces, denoted as I. Table I lists some notations that will be used in the following discussion. Chromatic number $\chi(G)$ is the least number of colors for graph $\mathrm{G}$ such that any two adjacent nodes are assigned with distinct colors. Clique number $\kappa(G)$ is the number of vertices in the largest clique in $\mathrm{G}$, whereas $\delta(G)$ is the number of vertices in smallest maximal clique in $\mathrm{G}$. In the following, we use the matrix form for the ease of presentation. $\mathcal{Q}$ is general form of the clique matrix; in SR-SC networks, its dimension is defined by the number of maximal cliques and the number of links; in MR-MC context, the number of links is replaced by the number of tuples. $\mathcal{F}$ is the flow assignment vector denoting the amount of flow over each link or tuple.

1) Sufficient condition in SR-SC context [3]: In SR-SC networks, the sufficient condition can be reached by corrected the necessary clique condition (8) with a constant $\alpha$ :

$$
\mathcal{Q F} \leq \alpha \times \mathcal{W}
$$

Where $\mathcal{W}$ is the capacity vector with all entries equal to $w$. Essentially, for any graph $G$, the constant $\alpha$ is the inverse of imperfection ratio $\operatorname{imp}(G)$ [19], defined as the supremum of the ratio between its chromatic number and its clique number. In the SR-SC context, $\alpha$ is 0.46 if the link conflict graph is modeled as a unit disk graph (UDG). The basic idea of proving sufficiency is to construct a feasible schedule policy. Specifically, by transforming the conflict graph to a new graph, the flow rate is homogeneous at each node, and the least colors (chromatic number) for the new graph is related to the clique size (clique number) of the new graph. The imperfection ratio is the suitable tool connecting these two parameters. More details can be found in [3]. We would like to emphasize that the above conclusion relies on a fundamental property that the geometric distance is the only one factor determining the interference relation in the SR-SC network, termed as geometric distance property. Specifically, if two links interfere with each other, the only reason is that they are located within the interference range of each other. While in the MR-MC context, two links can be active simultaneously on different channels even if they are close enough, which implies that geometric distance is not the unique factor determining interference in MR-MC networks.

2) Sufficient condition in MR-MC context: Though the geometric distance property is not the unique reason determining the interference in MR-MC networks, the scaled constant is still the inverse of imperfection ratio. The difficulty is that the method [19] designed to compute the imperfection ratio for the SR-SC network is not valid due to the disruptively different geometric property in the MR-MC network. It is challenging to compute imperfection ratio of MDCG directly. We instead aim to study its relation with that of the SR-SC network since those co-channel interference in SR-SC network also exists in the MR-MC context for a common network topology.

We develop a new technique to estimate the imperfection ratio of MDCG. The basic idea is to compute the imperfection ratio through a fundamental analysis of the chromatic number and the clique number of an MDCG. We construct two feasible cliques in an MDCG considering the co-channel interference and radio interface contention respectively, and estimate their sizes based on the clique number of SR-SC network. Their sizes can be viewed as the lower bound of clique number (i.e., the size of maximum clique). Regarding the chromatic number, we start from a feasible SR-SC schedule (expressed in chromatic number), and divide a time slot into several sub-slots to ensure an interference-free schedule in MR-MC context. In particular, some tuples associated with the same link need to be scheduled in different sub-slots due to either co-channel interference or the radio interface contention. The number of total required sub-slots can be viewed as the upper bound of least required time slots (i.e., chromatic number) in MRMC networks. The results show that the imperfection ratio of MDCG is closely related with that of SR-SC networks; in some cases, they are exactly the same. Moreover, the constant is also determined by number of radio interfaces $I$ and the total available channels $C$.

We first focus on the clique number. The interference can be classified as the co-channel interference and the radio contention. Considering these two types of conflicts, we can construct two types of cliques, providing the lower bound of the clique number $\kappa(M D C G)$.

Lemma 1: The clique number $\kappa(M D C G) \geq$ $\max \left(I^{2} \kappa(C G), I C \Delta(G)\right)$.

Proof: It is obvious that the size of any feasible clique we can enumerate is the lower bound of the clique number. We first consider the co-channel interference. Assume that the clique number of SR-SC conflict graph is $\kappa(C G)$ for a given topology, there are $\kappa(C G)$ links mutually interfering in G. We try to construct a clique in MDCG based on these $\kappa(C G)$ links. We focus on one channel, says channel $\tilde{c}$, out of $C$ available channels. Since each node has $I$ radios, one link 
can be split to $I^{2}$ tuples associated with channel $\tilde{c}$, and there are totally $I^{2} \kappa(C G)$ such tuples from these $\kappa(C G)$ links on channel $\tilde{c}$. It is easy to justify that any two of these $I^{2} \kappa(C G)$ tuples will interfere with each other since they share the channel $\tilde{c}$ and their associated links are located within each other's interference range. In other words, regardless of the number of channels, we could always find a clique in MDCG containing at least $I^{2} \kappa(C G)$ tuples.

Next, we consider the radio interface contention within the neighborhood of a node. Suppose node $u$ is the node with largest node degree $\Delta(G)$, and there is no doubt that these $\Delta(G)$ links form a clique under SR-SC environment. We concentrate on one specific radio interface of node $u$, says radio $\tilde{i}$, out of $I$ radios. Thus, each link between $u$ and one of its neighbors can be mapped to $I C$ tuples which are assigned with the radio interface $\tilde{i}$ on $u$. There are totally $I C \Delta(G)$ such tuples from $\Delta(G)$ original links connected to $u$. Obviously, these $I C \Delta(G)$ tuples pair-wisely conflict since they share the same radio interface $\tilde{i}$ on node $u$. It is ensured that these $I C \Delta(G)$ tuples form a clique in MDCG.

Based on above arguments, those two cliques imply the lower bound of clique number for MDCG, thus we have $\kappa(M D C G) \geq \max \left(I^{2} \kappa(C G), I C \Delta(G)\right)$ for any topology.

We next discuss the chromatic number $\chi(M D C G)$. Considering scheduling problem in the SR-SC context, the physical meaning of chromatic number is the least number of time slots $\left(T_{\min }\right)$ required for an interference-free schedule, such that each link will be assigned with at least one time slot. In MR-MC networks, the time slot is allocated to various tuples, we divide a time slot in SR-SC networks into several sub-slots for the tuples originated from one link. The basic idea is that given a feasible schedule in SR-SC networks, we can reveal the relationship between $\chi(M D C G)$ and $\chi(C G)$ by exploiting the parallel transmissions among tuples associated with one link. Toward this direction, we design two scheduling policies to estimate the required time slots, termed as scheduling mode 1 and scheduling mode 2, respectively. Each provides the upper bound of $\chi(M D C G)$ from different perspectives.

1) Scheduling Mode 1: With multiple radios and channels, we can naturally exploit the simultaneous transmissions which is not allowed in SR-SC networks. Given the chromatic number $\chi(C G)$ of SR-SC networks, we could guarantee an interference-free scheduling having $\chi(C G)$ time slots such that each link grabs at least one slot. It is worth noting that several non-interfering links may be active in the same time slot. There is no need to care about how many such links in each time slot, since there is no conflict between the tuples originated from different non-interfering links. In the MR-MC context, a link is mapped to $I^{2} C$ tuples. Intuitively, if the parallel transmission is not allowed for these tuples, we need $I^{2} C$ sub-slots for these tuples such that each tuple can be assigned with one sub-slot. In other words, for a time slot $t$ in SR-SC context, $I^{2} C$ sub-slots in MR-MC context can ensure an interference-free schedule for all tuples associated with the set of non-interfering links active in $t$. We therefore could construct an interference free schedule in MR-MC network, requiring $I^{2} C \chi(C G)$ time slots, and each tuple is assigned with at least one time slot. Obviously, this argument is quite loose since we do not consider the parallel transmissions among the $I^{2} C$ tuples originated from one link. In theory, there can be up to $I$ concurrent transmissions on distinct channels for the tuples originated from a link, since each node is equipped with $I$ radios and each link could be mapped to at most $I$ tuples without sharing any radio interface. We have following argument: If $I \leq C$, all the $I$ interface-free tuples originated from one link can be assigned with a common channel, and they can be active at the same time. Thus, the total amount of required time slots is: $\frac{I^{2} C \chi(C G)}{I}=I C \chi(C G)$; In the case of $I>C$, only $C$ out of $I$ tuples can be active in one time slot, occupying all $C$ channels. Consequently, the total required time slots is $\frac{I^{2} C \chi(C G)}{C}=I^{2} \chi(C G)$. In summary, under scheduling mode 1 , the chromatic number can be bounded as:

$$
\chi(M D C G)=T_{\min } \leq I \chi(C G) \max (I, C)
$$

2) Scheduling Mode 2: Scheduling mode 1 only takes the parallel transmissions over non-interfering links into account. It is straightforward, but not accurate enough. In fact, with multiple radios and channels, concurrent transmissions within an interference area is allowed, which will provide a more accurate estimation of required time slots. We have the definition:

Definition 1: If two links do not share any end point, then they are called separate links.

According to the definition, if two links have four disjoint end-points, then they are separate links and allowed to be active simultaneously through different channels regardless of their locations. The challenge is to estimate how many separate links within an interference area. Clique is the appropriate concept indicating such conflict information. We thus have the following lemma:

Lemma 2: There are at least $\frac{\delta(C G)}{2 \Delta(G)}$ separate links in a maximal clique.

Proof: Suppose there are $x$ separate links in a maximal clique $C L$ with size $\gamma$, we have $2 x$ disjoint nodes correspondingly, and their degrees are denoted as $d_{i}, i=1,2, \cdots, 2 x$. We have inequality $\sum_{i=1}^{2 x} d_{i} \geq \gamma$. Because one link is counted twice from node degree perspective, the left hand side is upper limit of number of links incident on these $2 x$ nodes. Note that this number is also the upper bound of the number of links in the clique $C L$. Since $x$ is the number of all the separate links in $C L$, that means any link belonging to clique $C L$ but outside these $x$ links must be incident on one of these $2 x$ nodes; Otherwise, either the link does not belong to $C L$ or we have $x+1$ separate links in $C L$. Further $d_{i} \leq \Delta(G), \forall i=1, \cdots, 2 x$, then $2 x \Delta(G) \geq \sum_{i=1}^{2 x} d_{i} \geq \gamma$. Therefore, we have:

$$
x \geq \frac{\gamma}{2 \Delta(G)} \geq \frac{\delta(C G)}{2 \Delta(G)}
$$


The second inequality relies on the fact that $\delta(C G)$ is the minimum maximal clique. Thus, we have the conclusion.

Lemma 2 describes the geometric property in the SRSC context, which enables us exploiting potential concurrent transmissions within a clique. The $\frac{\delta(C G)}{2 \Delta(G)}$ separate links could be split to $I \frac{\delta(C G)}{2 \Delta(G)}$ tuples, which do not share any radio interface. Ideally, if we have $I \frac{\delta(C G)}{2 \Delta(G)}$ channels, each tuple selects one distinct channel and all of them can be active during the same time slot. In general, if $I \frac{\delta(C G)}{2 \Delta(G)} \geq C$, that means only $C$ out of $I \frac{\delta(C G)}{2 \Delta(G)}$ tuples could be active at the same time because there are only $C$ available channels. And we apply the same argument for the total number of required time slots without concurrent transmission in scheduling mode 1 (i.e., $I^{2} C \chi(C G)$ ). Further, since $\kappa(C G) \geq \delta(C G)$, the chromatic number is:

$$
\begin{aligned}
& \text { if } I^{2} \kappa(C G) \geq 2 I C \Delta(G) \\
& \chi(M D C G)=T_{\min } \leq \frac{I^{2} C \chi(C G)}{C}=I^{2} \chi(C G)
\end{aligned}
$$

Obviously, the expression (12) under scheduling mode 2 is tighter than that (10) under mode 1 , since the number of radio interfaces is usually smaller than the number of channels. The essence is that we explore the parallel transmissions within a clique, which is not taken into account by mode 1 . Both estimate the upper bound of the least required time slots (i.e., chromatic number), which are expressed in terms of chromatic number of SR-SC networks. We have following theorem:

Theorem 3: In general, the imperfection ratio of MDCG is:

$$
\begin{aligned}
& i m p(M D C G)=\sup \frac{\chi(M D C G)}{\kappa(M D C G)} \\
& \leq \sup \frac{\chi(C G) \max (C, I)}{\max (I \kappa(C G), C \Delta(G))}
\end{aligned}
$$

Specially, if

$$
\begin{aligned}
& \kappa(C G) \geq \frac{2 C \Delta(G)}{I} \quad\left(\text { i.e., } I^{2} \kappa(C G) \geq 2 I C \Delta(G)\right) \\
& i m p(M D C G)=\sup \frac{\chi(M D C G)}{\kappa(M D C G)} \leq \sup \frac{I^{2} \chi(C G)}{I^{2} \kappa(C G)} \\
& =\sup \frac{\chi(C G)}{\kappa(C G)}=i m p(C G)
\end{aligned}
$$

Theorem 3 implies that the imperfection ratio of MR-MC network is closely related to that of SR-SC networks, and under certain condition they are the same. This is because the MR-MC network only incurs the radio interface contentions and shares those co-channel interference existing in the SRSC networks. This is a general conclusion for any topology, though we estimate the clique number and chromatic number assuming a specific topology. Now, we are ready to present the sufficient clique constraint:

Theorem 4: For MR-MC network, there is a feasible schedule for flow allocation $\mathcal{F}$ if

$$
\mathcal{Q F} \leq \beta \times \mathcal{W}
$$

Where $\beta$ is the inverse of imperfection ratio of MDCG given by theorem 3 .

Proof: Assume the entries of flow vector $\mathcal{F}$ are rational multiples of each other. We can find $T$, which is the smallest integer such that each entry $\mathcal{F}_{j}$ is integer multiples of $\frac{W}{T}$ for all entries in $\mathcal{F}$. For each $\mathcal{F}_{j}$, there is an integer $\mathcal{R}_{j}$ such that $\mathcal{F}_{j}=\mathcal{R}_{j} \times \frac{W}{T}$. We thus derive a vector $\mathcal{R}$ with element $\mathcal{R}_{j}$. We will construct a slotted time schedule for time period $T$.

We next transform MDCG to a new graph $\mathrm{MDCG}_{f}$, replacing a MDCG node $j$ by a clique consisting of $\mathcal{R}_{j}$ nodes. Each node in the clique replacing node $j$ needs a unique color. Further, the nodes in $\mathrm{MDCG}_{f}$ have homogeneous flow rates. If two nodes are adjacent in MDCG, all their components in the replaced cliques are adjacent in $\mathrm{MDCG}_{f}$, hence have unique colors. Let each color represent a time slot, we can ensure that two adjacent MDCG-nodes are assigned with different time slots. This implies that a coloring of $\mathrm{MDCG}_{f}$ implies a schedule of MDCG.

Note that the new $\mathrm{MDCG}_{f}$ is still MDCG, thus has all properties of MDCG. If we could prove the chromatic number of $\mathrm{MDCG}_{f}$ is at most $T$ (i.e., $\chi\left(M D C G_{f}\right) \leq T$ ), we would have a feasible schedule ensuring that each MDCG node is assigned with at least one slot without interference. We observe that $\mathcal{Q F} \leq \beta \times \mathcal{W}$ implies that $\mathcal{Q R} \leq \beta \mathcal{T}$, where $\mathcal{T}$ is a vector with all entries equal to $T$. Consequently, we have $\kappa\left(M D C G_{f}\right) \leq \beta T$ since clique number is the largest element of $\mathcal{Q R}$. Moreover, theorem 3 shows that $\chi\left(M D C G_{f}\right) \leq \frac{1}{\beta} \times \kappa\left(M D C G_{f}\right)$. We have $\chi\left(M D C G_{f}\right) \leq$ $\frac{1}{\beta} \times \kappa\left(M D C G_{f}\right) \leq T$. Therefore, we have a feasible schedule for any flow assignment $\mathcal{F}$ satisfying the sufficient condition (16).

Usually, the number of channels is larger than the number of radios (i.e., $C>I$ ), and the clique number is much larger than maximum node degree. Therefore, constant $\beta$ can be simplified to $\frac{I}{C} \frac{\kappa(C G)}{\chi(C G)}$. This clearly shows that the constant in MR-MC context is corrected by the ratio $\frac{I}{C}$ compared to the case in SR-SC context, which matches the conclusion in [15]. In the special case $\kappa(C G) \geq \frac{2 C \Delta(G)}{I}, \beta$ is exactly the same as the constant in the SR-SC context. Note that the sufficient condition (16) generates an $\beta$-approximation flow rate assignment compared to the optimal flow allocation. In other words, it could guarantee a capacity region no smaller than $\beta$ times the optimal capacity region.

\section{Distributed Algorithm to Compute Cliques}

For our purpose, we would like to compute maximal clique in a distributed and localized manner. Some algorithms [18], [20] are provided to generate cliques. However, they are either centralized or only suitable for unit disk graph in the SRSC context. We apply the straightforward method computing the maximal clique. Assume that each link is aware of its interfering links, then each link could compute the clique it belongs to based on this information. Because maximal clique containing link $l$ will not contain any other link outside the interference range of link $l$. Even in MR-MC context, two tuples will not conflict if the two links they are associated 


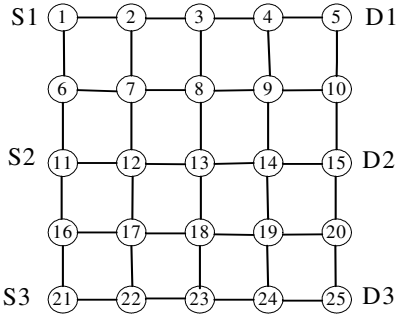

Fig. 3. Grid topology.

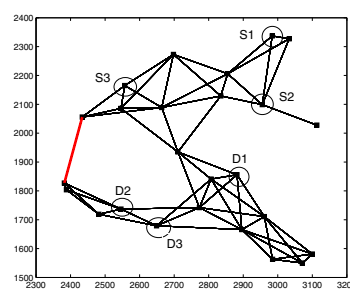

Fig. 4. Random topology.

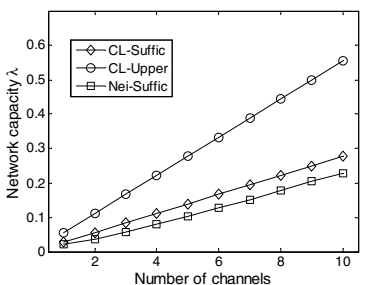

(a) Grid topology with 4 radios.

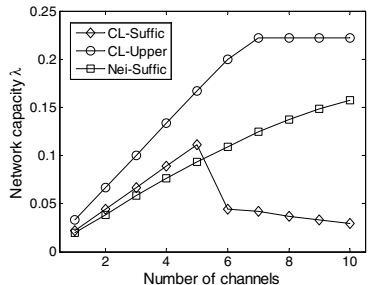

(b) Random topology with 2 radios. with are outside each other's interference range. Therefore, each tuple could compute all maximal cliques it belongs to with the knowledge of its interfering tuples.

Actually, once a tuple computes a set of maximal cliques it belongs to, it could independently check the sufficient condition. In particular, for each tuple $p$,

$$
\mathcal{Q}^{p} \mathcal{F}^{p} \leq \beta \times \mathcal{W}^{p}
$$

Where $\mathcal{Q}^{p}, \mathcal{F}^{p}$ and $\mathcal{W}^{p}$ are clique matrix indicating the maximal cliques the tuple $p$ belongs to, flow allocation vector and capacity vector for the tuples in $N(p)$.

\section{NUMERICAL RESULTS}

In this section, we present some numerical results to demonstrate the effectiveness of the neighborhood constraint and the sufficient clique constraint. To reveal the capacity region defined by different kinds of constraints, we formulate the multicommodity flow (MCF) problem, which incorporates some basic flow conservation constraints with different interference constraints. We consider two network topologies: 1) a grid topology as shown in Fig. 3, where every pair of nodes are separated by $200 \mathrm{~m}$; 2) a random topology as shown in Fig. 4 , with 25 nodes forming a connected network. There are 3 traffic flows in each topology. The source and destination nodes for flow $i(i=1,2,3)$ are denoted as $S_{i}$ and $D_{i}$ respectively. The transmission range and interference range is set to $250 \mathrm{~m}$ and $500 \mathrm{~m}$, respectively. For convenience of comparison, we assume that all physical link capacity $w_{u v}^{c}$ is one rate unit. Each flow has a rate demand of 3 rate units. The objective function of MCF formulation is $\lambda$ indicating the portion of flow requirements that can be supported by the network. All nodes have the same number of radio interfaces. We develop $\mathrm{C}$ codes to implement the algorithms presented in [1], and use CPLEX [21] to solve the optimization problems involved in our MCF formulation. We further denote the upper bound from clique constraint as CL-Upper, the solution based on sufficient clique constraint as CL-Suffic and the results from neighborhood constraint as Nei-Suffic. We also denote the MIS-based solution under the SIO-based computing as SIO-MIS [12] and the heuristic algorithms in [1] as static approximation (S-APP).

\section{A. Effectiveness of the Proposed Sufficient Constraints}

The effectiveness of the two sufficient constraints is shown in Fig. 5, illustrating the curve of $\lambda$ versus the number
Fig. 5. Performance of MDCG-based capacity computing.

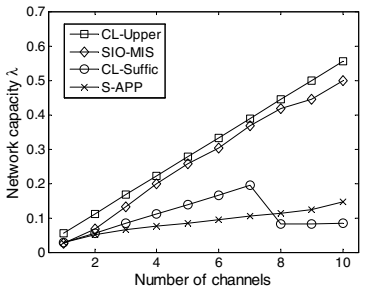

(a) Grid topology with 3 radios.

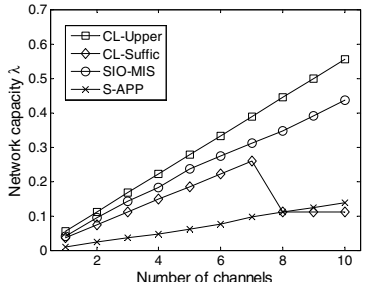

(b) Random topology with 3 radios.
Fig. 6. Comparison among feasible solutions.

of channels. In the left figure, the scaled clique constraint generates higher network capacity than that of neighborhood constraint as expected. The condition (14) is always satisfied with 4 radios and less than 10 channels in grid topology. Thus the achievable throughput of sufficient clique condition increases linearly as the clique constraint (8), and derives strictly half (i.e., $\beta=\frac{1}{2}$ ) of the clique upper bound. The clique number is 20 and the chromatic number is 40 , respectively. However, in random topology when each node is equipped with 2 radios, the condition (14) is satisfied only when the number of channels is no greater than 5. Correspondingly, the capacity of sufficient clique constraint begins degrading at 6 channels, since the scaled constant $\beta$ decreases according to theorem 4 . In this case, the neighborhood constraint outperforms the sufficient clique condition, which matches the conclusion about the achievable capacity region in [3], which claims that the neighborhood constraint leads to a larger capacity region than that of sufficient clique condition when the network is simple or there are few network flows.

\section{B. Comparison between Feasible Solutions}

We also compare the performance of those algorithms providing feasible solutions, including MIS-based computing [12], the S-APP algorithm [1] and the sufficient clique constraint. We also plot the clique upper bound as the performance benchmark in Fig. 6. We first justify that the sufficient clique condition indeed works out the feasible solution by following steps: 1) Solve the MCF formulation augmented with clique 
TABLE II

DYNAMIC CHANNEL SWAPPING FOR LINK $(3,12)$ IN RANDOM TOPOLOGY BASED ON CLIQUE SUFFICIENT CONSTRAINT

\begin{tabular}{l||c|c|c|c}
\hline $\begin{array}{l}\text { Channel/ } \\
\text { Radio-pair }\end{array}$ & $(1,1)$ & $(1,2)$ & $(2,1)$ & $(2,2)$ \\
\hline Channel 2 & 0 & 0 & 0.0058 & 0.0617 \\
\hline Channel 3 & 0 & 0.0158 & 0.1032 & 0 \\
\hline Channel 4 & 0.0058 & 0.0343 & 0.1389 & 0 \\
\hline Channel 5 & 0 & 0.1396 & 0 & 0.0542 \\
\hline
\end{tabular}

constraint. 2) Determine the scaled constant according to theorem 4, and scale down the clique based flow allocation derived in step 1.3) Modify the MIS-based MCF by removing the flow requirement and adding the scaled flow allocation as constraints, then solve the new MCF problem. The scaled flow allocation is feasible if the new MCF is solvable. In the experiment, we first utilize the clique based flow allocation as constraint, and there is no feasible schedule. This can be treated as evidence for the insufficiency of clique constraint (8). If we scale down the flow assignment by the constant $\beta$, the new MCF turns to be solvable, and we derive a feasible schedule. Table II shows the flow assignment for tuples associated with links $(3,12)$ (labeled as the red line in Fig.4) from the clique sufficient constraint in random topology, for instance of 2-interface node and 5 channels. The dynamic channel switching is achieved, utilizing four channels (channel 2, 3, 4 and 5). From Fig. 6, we observe that MIS-based solution is always better than the other two, since MIS computing is to provide optimal solution with all maximal independent sets involved. More interesting behavior is that the sufficient clique condition steadily outperforms the S-APP algorithm with no more than 7 channels for both topologies. When number of channels exceeds 7 , its performance sharply degrades and stops increasing even with more channels. The reason is the decreasing of the scaled constant. With no more than 7 channels, the condition (14) is satisfied for both topologies, so that the constant is fixed to $\frac{20}{40}$ and $\frac{40}{60}$ for grid and random topology, respectively. This also explains why the sufficient clique constraint linearly increases within this range. With more than 7 channels, condition (14) does not hold, and the constant is $\frac{1}{4}, \frac{2}{9}$ and $\frac{1}{5}$ for 8 -, 9- and 10-channel case in random topology, which is much smaller than $\frac{40}{60}$. Since the constant decreases with the increment of channels, the capacity achieved by the sufficient clique condition stops increasing, even below the S-APP algorithm. This explicitly implies that the sufficient clique constraint is conservative sometimes.

\section{CONCLUSION}

In this paper, we study the local sufficient rate constraints for flow allocation in MR-MC networks. We focus on those sufficient conditions that can be constructed and verified in distributed manner with local information. We first develop neighborhood constraint considering the conflicts within the interference neighborhood of a tuple. Furthermore, we construct a more accurate sufficient condition, by scaling the clique constraint with a constant. We particularly develop the new technique to compute the scaled constant by exploring the unique geometric property of MR-MC networks, and show that the constant is closely related to that in SR-SC networks. Under certain condition, they are exactly the same. We also analyze the efficiency ratio of these two constraints by theoretically computing the conflict degree and imperfection ratio, respectively. The scaled clique constraint defines a larger capacity region than the neighborhood constraint does. To the best of our knowledge, this is the first effort studying the distributed sufficient conditions considering the unique geometric property of MR-MC networks.

\section{REFERENCES}

[1] M. Alicherry, R. Bhatia, and L. Li, "Joint channel assignment and routing for throughput optimization in multi-radio wireless mesh networks," in Proc. ACM MobiCom, Aug. 2005, pp. 58-72.

[2] M. Kodialam and T. Nandagopal, "Characterizing the capacity region in multi-radio multi-channel wireless mesh networks," in Proc. ACM MobiCom, Aug. 2005, pp. 73-87.

[3] R. Gupta, J. Musacchio, and J. Walrand, "Sufficient rate constraints for QoS flows in ad-hoc networks," in ACM Ad Hoc Networks, vol. 5, issue. 4, May. 2007, pp. 429-443.

[4] A. Raniwala and T.-C. Chiueh, "Architecture and algorithms for an IEEE 802.11-based multi-channel wireless mesh network," in Proc. IEEE INFOCOM, Mar. 2005, pp. 2223-2234.

[5] P. Kyasanur and N. H. Vaidya, "Routing and link-layer protocols for multi-channel multi-interface ad hoc wireless networks," SIGMOBILE Mobile Computing and Communications Review, vol. 10, no. 1, pp. 3143, Jan. 2006.

[6] L. Gao, X. Wang and Y. Xu, "Multi-radio Channel Allocation in Multihop Wireless Networks," IEEE Transactions on Mobile Computing., vol 8, no. 11, pp: 1454-1468, Nov 2009.

[7] A. Raniwala, K. Gopalan, and T. Chiueh, "Centralized algorithms for multi-channel wireless mesh networks," ACM Mobile Computing and Communications Review, vol. 8, no. 2, pp. 50-65, Apr. 2004.

[8] L. Gao and X. Wang, "A Game Approach for Multi-Channel Allocation in Multi-Hop Wireless Networks," in Proc. ACM MobiHoc, May 2008, Hong Kong.

[9] K. Jain, J. Padhye, V. Padmanabhan, and L. Qiu, "Impact of interference on multi-hop wireless network performance," in Proc. ACM MobiCom, 2003, pp. 66-80.

[10] V. S. Anil Kumar, M. V. Marathe, and S. Parthasarathy, "Algorithmic aspects of capacity in wireless neworks," in Proc. ACM SIGMETRICs, 2005, pp. 133-144.

[11] K. N. Ramachandran, E. M. Belding, K. C. Almeroth, and M. M. Buddhikot, "Interference-aware channel assignment in multi-radio wireless mesh networks," in Proc. IEEE INFOCOM, 2006.

[12] H. Li, Y. Cheng, C. Zhou, and P. Wan, "Multi-dimensional conflict graph based computing for optimal capacity in MR-MC wireless networks", in Proc. IEEE ICDCS, June, 2010.

[13] P. Gupta and P. R. Kumar, "The cpacity of wireless networks," IEEE Trans. Inform. Theory, vol. 46, no. 2, pp. 388-404,2000.

[14] J. Li, C. Blake, D. De Couto, H. Lee and R. Morris , "Capacity of Ad Hoc Wireless Networks," in Proc. ACM MobiCom, Jul. 2001, pp. 61-69.

[15] P. Kyasanur and N. H. Vaidya, "Capacity of multi-channel wireless networks: Impact of number of channels and interfaces," in Proc. ACM MobiCom, Aug. 2005, pp. 43-57.

[16] X. Lin and S. Rasool, "A distributed joint channel-assignment, scheduling and routing algorithm for multi-channel ad hoc wireless networks," in Proc. IEEE INFOCOM, May 2007, pp. 1118-1126.

[17] P. Chaporkar, K. Kar, and S. Sarkar, "Throughput Guarantees in Maximal Scheduling in Wireless Networks," in Proc. 43rd Annual Allerton Conference, September 2005.

[18] R. Gupta, J. Walrand and O. Goldschmidt, "Maximal cliques in unit disk graphs: polynomial approximation," in Proc. INOC, Mar. 2005.

[19] S. Gerke and C. McDiarmid, "Graph imperfection I," Journal of Combinatorial Theory, Series B 83 (2001) 58C78.

[20] R. Negi and A. Rajeswaran, "Physical layer effect on MAC performance in ad-hoc wireless networks," in Proceedings Communications, Internet and Information Technology CIIT, 2003.

[21] Ilog cplex suite, http://www.ilog.com/products/cplex/ 